\title{
Anomalous Anisotropy in the Explosion of Rare-Gas Clusters Irradiated with Intense Few-cycle Laser Pulses
}

\author{
E. Skopalová, Y. C. El-Taha, A. Zaïr, M. Hohenberger, \\ J. W. G. Tisch, R. A. Smith, and J. P. Marangos \\ Department of Physics, Imperial College London, \\ London, SW7 2AZ, United Kingdom
}

(Dated: November 26, 2009)

\begin{abstract}
We have experimentally observed anisotropic ion emission from Xe and Ar clusters subject to intense ultra-short ( $\sim 30 \mathrm{fs}$ ) near-infrared laser pulses, with up to $80 \%$ more energetic ions emitted in the perpendicular direction to the laser polarisation than in the parallel direction. Our pulse duration investigation in the range of $8-160$ fs revealed that this anisotropy is present in the interaction for a specific range of pulse durations. Treating electrons inside the unexpanded cluster as a harmonic oscillator qualitatively demonstrates how the intracluster electric field can result in an ion emission anisotropy consistent with our observations. Our observations and modelling therefore give direct access to the dynamics that will be present in the first few cycles of an intense laser field interacting with any nanoscale dielectric.
\end{abstract}


The interaction of intense $\left(>10^{15} \mathrm{~W} \mathrm{~cm}^{-2}\right)$ laser pulses with rare-gas clusters can give rise to energetic particles, mainly due to the enhanced energy absorption by the cluster medium [1, 2]. Fast highly-charged ions [3-6], electrons with energies in the keV range $[7,8]$ and energetic x-rays [9-11] have been observed after explosions of clusters subject to strong laser fields. Most of these earlier experiments were performed with pulses of durations $\tau$ of $100 \mathrm{~s}$ of fs, which is comparable to a typical cluster expansion time $t_{E}[2,11]$. In this regime, which we refer to as "adiabatic", the laser plasma continues to act on a timescale long enough for significant ion motion to occur. In the adiabatic regime, details of the laser-cluster interaction near the onset of irradiation are masked by subsequent events. With shorter laser pulses becoming widely available, clusters can be exposed to pulses with $\tau \ll t_{E}$, referred to as the "non-adiabatic" regime. In this regime, ions are effectively frozen during the laser pulse and additional details of the fundamental processes of the laser-cluster interaction can be obtained. This provides new insight into the early stages of a strong laser field interacting with a nanoscale dielectric.

Cluster explosions are driven primarily by two forces [12]: Coulomb repulsion between charged ions, and hydrodynamic pressure originating from a hot electron plasma. The relative importance of these forces depends on the laser and cluster parameters. Both limiting cases, pure Coulomb explosion [13] and pure hydrodynamic explosion [11], lead to a completely isotropic cluster disintegration. However, several authors have reported anisotropic cluster explosions with more energetic ions emitted along the laser polarisation axis $[4-7,14,15]$. These experiments were performed with a range of cluster species and sizes, mostly in the adiabatic regime. The anisotropy has been explained by several different mechanisms. In the hydrodynamic expansion of large Xe clusters [7, 14] a surface polarisation pressure with $\cos ^{2} \theta$ angular dependence is believed to be responsible for the anisotropic cluster expansion $(\theta$ is the angle between the laser polarisation axis and the ion emission direction). In the case of $\mathrm{Ar}$ and $\mathrm{N}_{2}$ clusters exploding predominantly by Coulomb forces $[4,5]$ the asymmetry of highly energetic ions has been interpreted using a "charge-flipping" mechanism resulting from a time-dependent ion charge state $[5,16]$, and the presence of higher charge states at $\theta=0^{\circ}$ predicted by PIC simulations [12]. Anisotropic explosions of hydrogen clusters [15] have been explained by partial stripping of the cluster and resulting oscillations of the electron cloud across the cluster [17].

In this Letter, we study angularly resolved ion emission from Xe and Ar clusters irradiated 
by laser pulses with durations in the range of $8-160 \mathrm{fs}$, covering the non-adiabatic and the adiabatic regime. We demonstrate for the first time that, for a certain range of pulse durations (specific to the cluster type, but in our case around 10-30 fs, in the non-adiabatic regime), more energetic ions are emitted from clusters in the direction perpendicular to the laser polarisation axis, which is in contrast to earlier measurements $[4-7,14,15]$. We explain the observed anisotropy with an electrostatic model in which the cycle-averaged intracluster electric field is calculated. It shows that the field at $\theta=90^{\circ}$ can be enhanced due to collective electron oscillations inside the cluster. Moreover, we demonstrate that a continuous transition of the interaction from the non-adiabatic to the adiabatic regime is directly mapped to the ion angular distribution. This transition can be universal to any nanoscale object.

Our experiment was performed by irradiating a low density cluster beam with a laser beam with laser pulse durations ranging from 8 fs up to 160 fs. The laser used was a $1 \mathrm{kHz}$ Ti:sapphire CPA system (KML Inc.), delivering approximately transform-limited pulses with a central wavelength of $790 \mathrm{~nm}$ and duration of $28 \mathrm{fs}$. Longer pulses were produced by detuning a compressor grating pair. An energy of $1 \mathrm{~mJ}$ per pulse was used for experiments with pulse durations of $28-160$ fs. Shorter pulses were produced using a differentially pumped hollow fibre and chirped mirrors compression system. The pulse duration was varied in the range of $8-21 \mathrm{fs}$ by adjusting the gas pressure inside the fibre [18]. The energy per pulse from the hollow fibre system was $\sim 300 \mu \mathrm{J}$. The laser was focused into the cluster beam with an off-axis parabolic mirror to peak intensities of $4 \times 10^{15} \mathrm{~W} \mathrm{~cm}^{-2}$ for the $28 \mathrm{fs}$ pulse, and $5 \times 10^{15} \mathrm{~W} \mathrm{~cm}^{-2}$ for the $8 \mathrm{fs}$ pulse. The temporal profile of the laser pulses was diagnosed with a SHG-FROG [19]. Xe and Ar clusters were produced by expansion of room temperature pressurised gas through a solenoid pulsed valve with a $0.5 \mathrm{~mm}$ nozzle [20]. A skimmer with an aperture of $1 \mathrm{~mm}$ was placed $10 \mathrm{~cm}$ below the nozzle to collimate a cluster beam with density of $\sim 10^{14}$ atoms $/ \mathrm{cm}^{3}$. Mean cluster sizes were estimated from the Hagena's scaling law [21]. Ions emitted upon cluster explosions were detected with a microchannel plate in the direction perpendicular to the laser and cluster beams and their energies determined from field-free time of flight. The polarisation of the laser was rotated by a half-wave plate placed before the focusing optics in order to measure angularly dependent ion emission.

Measured ion energy spectra of $\mathrm{Xe}_{2600}$ clusters irradiated with 28 fs pulses (in the non- 

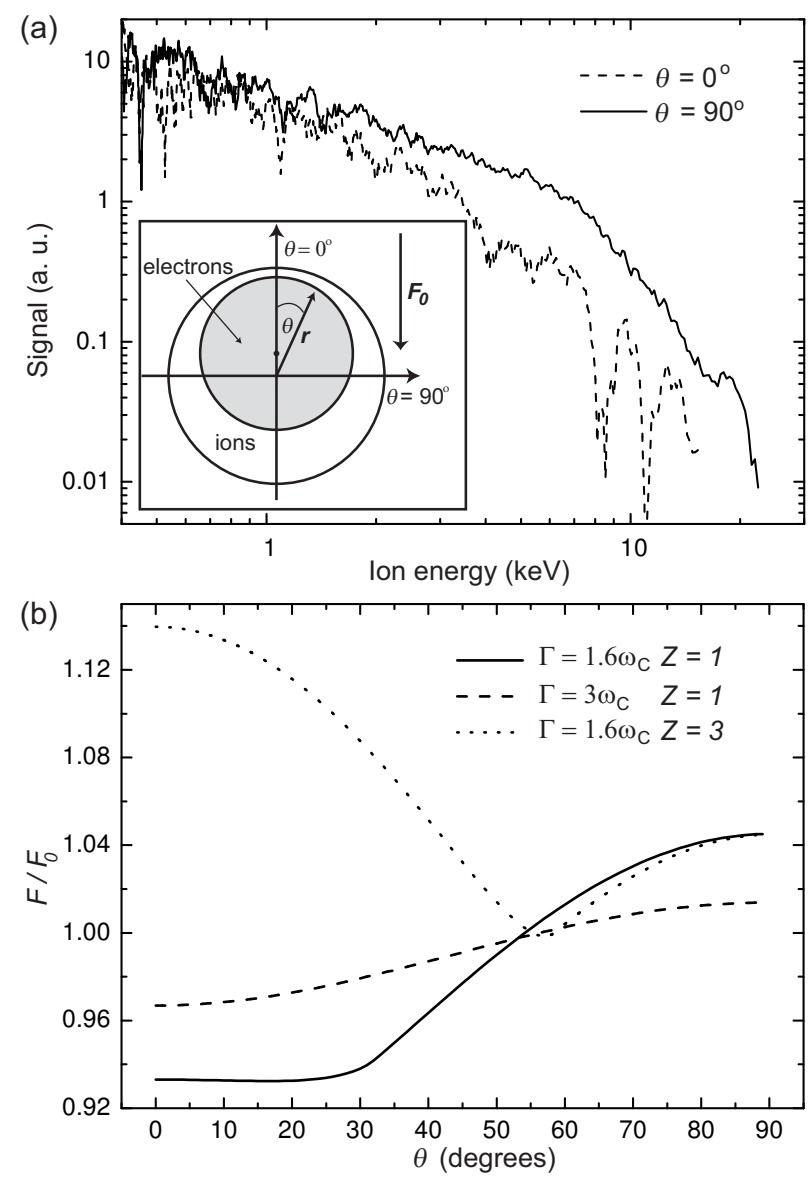

FIG. 1. (a) Ion energy spectra of $\mathrm{Xe}_{2600}$ clusters measured at $\theta=0^{\circ}$ (dashed) and $\theta=90^{\circ}$ (solid). Pulse duration $28 \mathrm{fs}$, peak intensity $4 \times 10^{15} \mathrm{~W} \mathrm{~cm}^{-2}$. Inset - geometry of our electrostatic model. (b) Calculated cycle-averaged electric field at the surface of $\mathrm{Xe}_{2600}$ cluster for different $\Gamma$ and $Z$. Electric field $F_{0}$ in the calculation corresponds to intensity of $4 \times 10^{15} \mathrm{~W} \mathrm{~cm}^{-2}$.

adiabatic regime) with peak intensity of $4 \times 10^{15} \mathrm{~W} \mathrm{~cm}^{-2}$ are shown in Fig. 1a. More energetic ions are emitted in the direction perpendicular to the laser polarisation. The mean ion energies are $\sim 3.3 \mathrm{keV}$ for $\theta=90^{\circ}$ and $\sim 1.8 \mathrm{keV}$ for $\theta=0^{\circ}$. We have also measured electron emission from clusters under the same conditions, and observed preferential electron emission in the direction parallel to the laser polarisation axis, i.e. in the orthogonal direction to the highly-energetic ion emission. The anticorrelation between the directions of electron and highly-energetic ion emission suggests that their origin is decoupled, i.e. energetic ions are not accelerated by hydrodynamic pressure. On the contrary, electrostatic forces are seemingly more significant in determining the trajectories of the most energetic ejected ions, particularly of ions emitted in the direction perpendicular to the laser polarisation axis. 
Hydrodynamic pressure can play a role in acceleration of ions with lower energies in the isotropic part of the ion spectrum. We have observed similar behaviour in explosions of $\mathrm{Ar}$ clusters with comparable sizes as Xe clusters under the same conditions of laser irradiation.

We qualitatively explain the anisotropy with an electrostatic model calculating the intracluster electric field, averaged over one cycle of $\mathrm{cw}$ irradiation, that drives Coulomb expansion of the cluster. The simplifications made in our model can be justified by molecular dynamics simulations [22] that have shown that a simplified description of collectively oscillating electrons inside the cluster is in good agreement with predictions of molecular dynamics codes. Moreover, the model presented here is similar to the analytical model in Ref. [17] that has been successfully applied to explain anisotropic explosions of hydrogen clusters [15]. We treat the cluster as a 3D homogeneously charged sphere with an average ionic charge state $Z$ (inset in Fig. 1a). We estimate the fraction of electrons that leave the cluster by lowering of the cluster potential barrier by the laser electric field [23]. Electrons remaining inside the cluster are assumed to form a sphere with density equal to the ionic charge density, hence the interior of the electron sphere remains neutral. The electron sphere is described as a harmonic oscillator driven by the laser electric field $F_{0} \sin (\omega t)$ and damped by various processes, including collisions and additional ionisation [22], that are included in the damping rate $\Gamma$. $\Gamma$ is chosen to be on the order of cluster eigenfrequency $\omega_{C}$ in accordance with predictions of molecular dynamics simulations [22]. The total electric field of ion and electron spheres is calculated and averaged over one laser period. It is anisotropic because of oscillations of the electron sphere in the laser field direction, though it is axially symmetric. The cycle-averaged field $F$ at the cluster surface can have a maximum either at $\theta=0^{\circ}$ or at $\theta=90^{\circ}$, depending on the initial cluster and laser conditions and the choice of free parameters of the model: charge state $Z$ and damping rate $\Gamma$. The role of the chosen parameters can be inferred from Fig. 1b, that shows calculated electric field at the surface of a $\mathrm{Xe}_{2600}$ cluster for different $Z$ and $\Gamma$. We show the electric field at the cluster surface as this drives the ejection of the most-energetic ions [5, 13]. A notable anisotropy in the electric field appears in the case of $Z=1$ and $\Gamma=1.6 \omega_{C}$ (solid line) where the electric field for $\theta=90^{\circ}$ is $12 \%$ larger than for $\theta=0^{\circ}$. The angular profile of the calculated electric field changes when the parameters are varied, e.g. the increase of $\Gamma$ leads to the decrease of the amplitude of oscillations of the electron sphere, making the field more isotropic (dashed line). Increasing charge state $Z$ eventually leads to an electric field profile with a maximum 


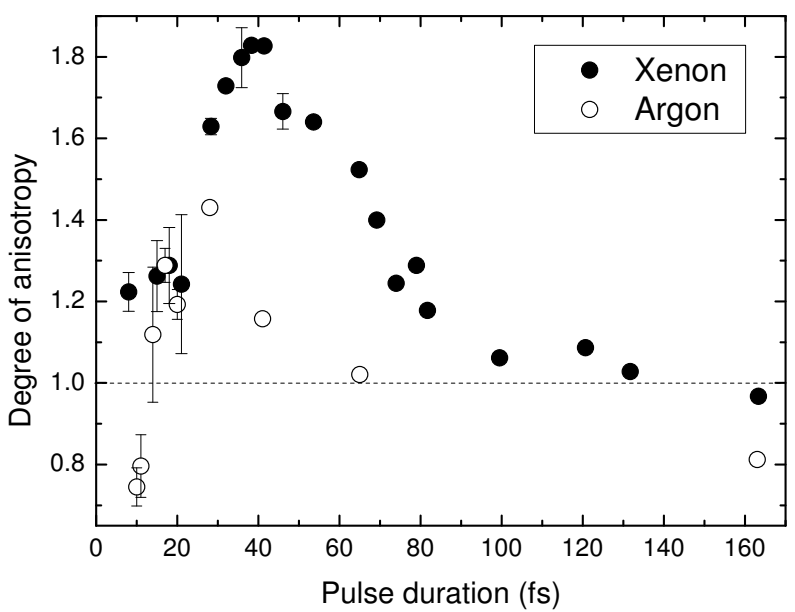

FIG. 2. Measured degree of anisotropy $E_{90^{\circ}} / E_{0^{\circ}}$ determined from mean ion energies of $(\bullet) \mathrm{Xe} \sim 14000$ and (॰) $\operatorname{Ar}_{\sim 14000}$ clusters as a function of pulse duration. Maximum anisotropy degree is $E_{90^{\circ}} / E_{0^{\circ}}=$ 1.83 for Xe clusters irradiated by a 40 fs pulse

at $\theta=0^{\circ}$ (dotted line). The dependence of the electric field angular profile on the cluster size and density and on the laser intensity is more gradual. Overall, the model shows that the electric field at the cluster surface can have a maximum at position $\theta=90^{\circ}$, thus it can induce an anisotropic cluster explosion with more energetic ions ejected in the direction perpendicular to the laser polarisation axis. The mechanism described here will play a role in cluster explosions for a sufficiently short pulse, as will be discussed later.

In order to track the changes in the ion anisotropy in different interaction regimes, we have performed an extensive pulse duration investigation in the range of $8-160 \mathrm{fs}$. Ion emission from Xe and Ar clusters has been measured in directions $\theta=0^{\circ}$ and $\theta=90^{\circ}$ as a function of pulse duration, and the mean ion energies were determined from each ion spectrum. In order to quantify the observed anisotropy we define a degree of anisotropy $E_{90^{\circ}} / E_{0^{\circ}}$, the ratio of mean ion energies in the two orthogonal directions. Values of $E_{90^{\circ}} / E_{0^{\circ}}>1$ correspond to the new anisotropy with more energetic ions emitted for $\theta=90^{\circ}$. The degree of anisotropy of $\mathrm{Xe}_{\sim 14000}$ and $\mathrm{Ar}_{\sim 14000}$ clusters for the whole range of pulse durations studied is presented in Fig. 2. Although intensities from the hollow fibre and from the laser system are different $\left(4 \times 10^{15} \mathrm{~W} \mathrm{~cm}^{-2}\right.$ for the 28 fs pulse, $5 \times 10^{15} \mathrm{~W} \mathrm{~cm}^{-2}$ for the $8 \mathrm{fs}$ pulse $)$, results obtained with these two light sources can be directly compared, because measurements have shown only a small variation of the anisotropy degree with intensity.

In the few-cycle regime, ion emission from Xe clusters is anisotropic with $E_{90^{\circ}} / E_{0^{\circ}} \approx 1.25$ 
with the anisotropy persisting for pulse durations of 8-100 fs. This dependance on the pulse duration can be explained by the fact that heavy Xe ions essentially do not move during the laser pulse. On the other hand, ion emission from lighter Ar clusters in the few-cycle laser fields undergoes rapid changes when the pulse duration is increased from $\sim 10$ fs to $\sim 20$ fs. In this case, for the shortest pulse durations, the anisotropy degree drops below 1 .

The anisotropy degree both for Ar and Xe clusters decreases and eventually drops below 1 as the pulse duration increases, hence the anisotropy is reversed back to the well-known case. This fact indicates a competition between physical processes driving cluster expansions on different time-scales. For longer pulse durations in the adiabatic regime, the anisotropy with degree of $E_{90^{\circ}} / E_{0^{\circ}}<1$ can be explained by mechanisms such as surface polarisation acceleration [7] and the "charge-flipping" process [5, 16]. However, these mechanisms are not expected to be significant in the non-adiabatic regime for the following reasons. Surface polarisation pressure will not be resonantly enhanced when the cluster does not expand. The "charge-flipping" force will be less important for shorter pulses because it acts on ions for a smaller number of laser cycles. Therefore, other processes of ion acceleration start to prevail in the non-adiabatic regime. Our electrostatic model shows that the intracluster electric field can have $\sim 12 \%$ higher values at $\theta=90^{\circ}$ than at $\theta=0^{\circ}$. Although only the cycle-averaged electric field is calculated and pulse duration is not considered explicitly in the calculation, a dependence on the pulse duration can be inferred. One can expect that a sufficiently long pulse will deposit more energy into the cluster during the cluster expansion and thus create higher charge states [10]. The degree of anisotropy in our model decreases for higher ionic charge states, therefore it should decrease with increasing pulse duration. Moreover, the damping rate can change substantially for different pulse durations, leading to significant changes in the electric field profile (Fig. 1b).

We find that the transition of anisotropy degree $E_{90^{\circ}} / E_{0^{\circ}}$ from $>1$ to $<1$ occurs at a specific pulse duration for each atom species and cluster size investigated. We designate this pulse duration the "transition time" $t_{A}$ and analyse it in more detail. The experimentally determined transition time $t_{A}$ for each cluster type can be compared to the cluster explosion time $t_{E}$. We estimate characteristic cluster explosion time as the time needed for a cluster to double its initial radius $R_{0}$ assuming ions exploding hydrodynamically at a plasma sound speed [11]: $t_{E}=R_{0} \sqrt{m_{i} / Z k T_{e}}$, where $Z$ is the ion charge state, $k T_{e}$ is the electron temperature and $m_{i}$ is the ion mass. The assumption of hydrodynamic expansion should 


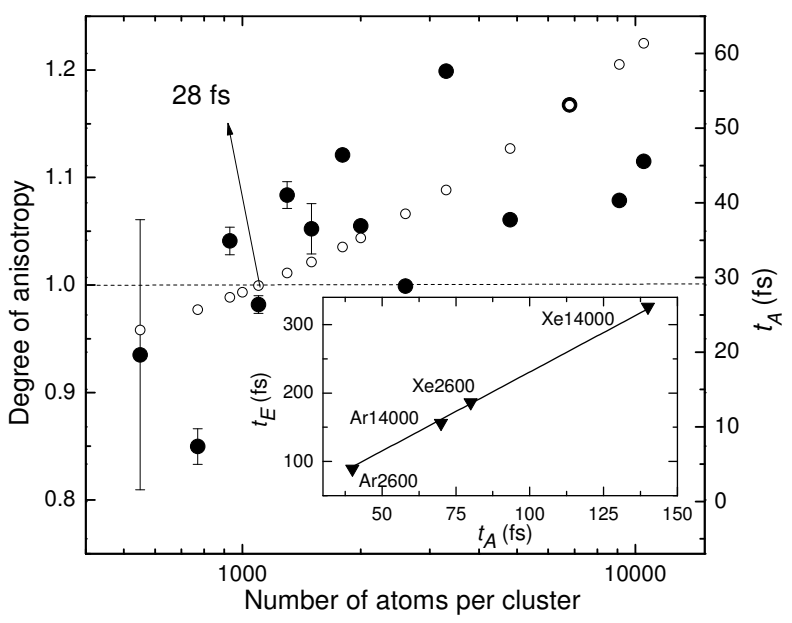

FIG. 3. (•) Left axis - measured anisotropy degree as a function of size for Ar clusters, irradiated with 28 fs pulses with peak intensity of $4 \times 10^{15} \mathrm{~W} \mathrm{~cm}^{-2}$. (o) Right axis - estimated transition time $t_{A}$. Inset - relation between estimated explosion time $t_{E}$ and experimentally determined transition time $t_{A}$ for Xe and Ar clusters containing 2600 and 14000 atoms. Linear fit $t_{E}=a t_{A}$ with $a=2.3$ is also shown.

be reasonably valid in the adiabatic regime. The relation between the estimated expansion time $t_{E}$, averaged over a realistic log-normal cluster size distribution [24], and experimentally determined transition time $t_{A}$ for four different clusters is presented in the inset of Fig. 3. A strong correlation between the expansion time and the transition time can be seen: $t_{E}=a t_{A}$ with $a=2.3$. We note that for calculation of $t_{E}$, values of $Z=1$ and $k T_{e}=500 \mathrm{eV}$ have been used, though the choice of these parameters only weakly influences the correlation between $t_{E}$ and $t_{A}$. Therefore, the change in ion anisotropy (quantified by $t_{A}$ ) is directly related to the transition of the interaction from the non-adiabatic to the adiabatic regime (represented by $t_{E}$ ). We can compare the experimentally determined anisotropy degree of Ar clusters of different sizes with predictions based on estimation of transition times (Fig. 3). Estimated transition times $t_{A}=t_{E} / a$ for Ar clusters are shown as open circles in Fig. 3. The calculated transition time $t_{A}$ increases with cluster size and has a value of 28 fs for Ar cluster with 1000 atoms. This estimation means that Ar clusters containing less than 1000 atoms irradiated with pulses of duration $\tau=28 \mathrm{fs}$ are in the adiabatic regime, because in this case $t_{A}<\tau$, so we predict explosions to be anisotropic with $E_{90^{\circ}} / E_{0^{\circ}}<1$. On the other hand, Ar clusters with more than 1000 atoms under 28 fs irradiation are expected to be in the non-adiabatic regime (because $t_{A}>\tau$ ), therefore they are expected to explode with 
anisotropy degree of $E_{90^{\circ}} / E_{0^{\circ}}>1$. This prediction can be compared with experimentally determined anisotropy degree of Ar clusters irradiated with 28 fs pulses (solid circles in Fig. 3). The agreement between estimation and experimental results further confirms the direct connection between the anisotropy degree and the cluster expansion regime.

In conclusion, we have observed anisotropic cluster explosions with significantly more energetic ions emitted in the direction perpendicular to the laser polarisation axis. We interpret this anisotropy in terms of Coulomb acceleration of the energetic ions by the electric field enhanced at $\theta=90^{\circ}$ due to collective electron oscillations. Ion acceleration mechanisms in the few-cycle regime need further investigation. We have found that for very short laser pulses, or in the early cycles of longer pulses, the internal collective electron oscillation driven by the field occurs within an initially fixed frame of ions. The anisotropic ion energy is a direct signature of this early phase of the laser matter interaction which is masked if longer pulses are used. This behaviour will be general to all nanoscale dielectrics.

This work is supported by the EPSRC. The technical assistance of Peter Ruthven and Andy Gregory is gratefully acknowledged.

[1] T. Ditmire, R. A. Smith, J. W. G. Tisch, and M. H. R. Hutchinson, Phys. Rev. Lett. 78, 3121 (1997).

[2] J. Zweiback, T. Ditmire, and M. D. Perry, Phys. Rev. A 59, R3166 (1999).

[3] T. Ditmire, J. W. G. Tisch, E. Springate, M. B. Mason, N. Hay, R. A. Smith, J. Marangos, and M. H. R. Hutchinson, Nature 386, 54 (1997).

[4] V. Kumarappan, M. Krishnamurthy, and D. Mathur, Phys. Rev. Lett. 87, 085005 (2001).

[5] M. Krishnamurthy, D. Mathur, and V. Kumarappan, Phys. Rev. A 69, 033202 (2004).

[6] M. Hirokane, S. Shimizu, M. Hashida, S. Okada, S. Okihara, F. Sato, T. Iida, and S. Sakabe, Phys. Rev. A 69, 063201 (2004).

[7] V. Kumarappan, M. Krishnamurthy, and D. Mathur, Phys. Rev. A 66, 033203 (2002).

[8] Y. L. Shao, T. Ditmire, J. W. G. Tisch, E. Springate, J. P. Marangos, and M. H. R. Hutchinson, Phys. Rev. Lett. 77, 3343 (1996).

[9] A. McPherson, B. D. Thompson, A. B. Borisov, K. Boyer, and C. K. Rhodes, Nature 370, $631(1994)$. 
[10] F. Dorchies, T. Caillaud, F. Blasco, C. Bont, H. Jouin, S. Micheau, B. Pons, and J. Stevefelt, Phys. Rev. E 71, 066410 (2005).

[11] T. Ditmire, T. Donnelly, A. M. Rubenchik, R. W. Falcone, and M. D. Perry, Phys. Rev. A 53, 3379 (1996).

[12] C. Jungreuthmayer, M. Geissler, J. Zanghellini, and T. Brabec, Phys. Rev. Lett. 92, 133401 (2004).

[13] I. Last and J. Jortner, J. Chem. Phys. 121, 3030 (2004).

[14] E. Springate, N. Hay, J. W. G. Tisch, M. B. Mason, T. Ditmire, M. H. R. Hutchinson, and J. P. Marangos, Phys. Rev. A 61, 063201 (2000).

[15] D. R. Symes, M. Hohenberger, A. Henig, and T. Ditmire, Phys. Rev. Lett. 98, 123401 (2007).

[16] K. Ishikawa and T. Blenski, Phys. Rev. A 62, 063204 (2000).

[17] B. N. Breizman, A. V. Arefiev, and M. V. Fomyts'kyi, Phys. Plasmas 12, 056706 (2005).

[18] J. S. Robinson, C. A. Haworth, H. Teng, R. A. Smith, J. P. Marangos, and J. W. G. Tisch, Appl. Phys. B 85, 525 (2006).

[19] D. J. Kane and R. Trebino, IEEE J. Quantum Electron. 29, 571 (1993).

[20] R. A. Smith, T. Ditmire, and J. W. G. Tisch, Rev. Sci. Instrum. 69, 3798 (1998).

[21] O. F. Hagena, Rev. Sci. Instrum. 63, 2374 (1992).

[22] U. Saalmann and J.-M. Rost, Phys. Rev. Lett. 91, 223401 (2003).

[23] I. Last and J. Jortner, Phys. Rev. A 60, 2215 (1999).

[24] K. J. Mendham, N. Hay, M. B. Mason, J. W. G. Tisch, and J. P. Marangos, Phys. Rev. A 64, 055201 (2001). 